\title{
Cytogenetic and Morpho-Agronomic Study of Some Gamma Irradiated Cotton (Gossypium hirsutum L.) Cultivars
}

\author{
Masoud Sheidai ${ }^{1, *}$ and Mahrooz Dezfolian ${ }^{2}$ \\ ${ }^{1}$ Faculty of Biological Sciences, Shahid Beheshti University, Evin, Tehran, Iran \\ ${ }^{2}$ Genetic Department, Islamic Azad University, Tehran, Iran
}

Received November 22, 2007; accepted December 7, 2007

\begin{abstract}
Summary A cytogenetic and morphometric study was performed on 5 M4 tetraploid cotton cultivars (Gossypium hirsutum L.). The chromosome pairing and chiasma frequency as well as meiotic abnormalities were compared among the genotypes studied. Meiotic abnormalities including cytomixis, formation of laggard chromosome and stickiness may be responsible for the reduction in pollen fertility and abnormal pollen grain formation in cotton cultivars studied. The cultivars studied differed significantly in their cytogenetic and morpho-agronomic characteristics indicating their genomic differences, which may be used in cotton breeding.
\end{abstract}

Key words Cotton, Cytogenetics, Cytomixis.

Tetraploid cotton (Gossypium hirsutum) with the genome constitution $2(\mathrm{AD})_{1}(2 n=52)$ along with $G$. barbadense dominate the world cotton production. The genomes of G. hirsutum individually are referred to as $\mathrm{A}_{\mathrm{h}}$ and $\mathrm{D}_{\mathrm{h}}$ and their chromosomes as $\mathrm{H} 1-\mathrm{H} 13$ and $\mathrm{H} 14-\mathrm{H} 26$, respectively. Mutation breeding using gamma irradiation has been used extensively in upland cotton leading to improvement in quantitative characters, insensitivity against the photoperiod, earliness, yield and oil content. Such studies have shown the occurrence of cytogenetic abnormalities and phenotypic changes in mutants (Abo-Hegazi and Shaheen 1988).

Cotton is considered as one of the most important crop plants in Iran, cultivated in various regions of the country. Continuous cultivation of the same genotypes may bring about genetic erosion in the long term, therefore study of the available genetic variability as well as introducing the new ones is of importance. The present report considers the cytogenetic and morpho-agronomic study of some gamma irradiated cotton cultivars obtained after M4 generation and extensive selection on phenotypic/agronomic characters considered to be stable in their phenotypic and genetic characteristics.

\section{Materials and methods}

Seeds of cotton cultivars namely Varamin, Shirpan, Tashkand and Sahel were treated with 150, 200, 250 and 300 grays (Gy) of gamma rays using Cobalt 60. Plants were raised from M1 to M4 by selfing and selection was performed based on important agronomic characters like earliness, lint quality and yield. The irradiated lines obtained are named Var-76, T-300, C-250, c-200 and S-150 accordingly. The plants were cultivated in three rows of $10 \mathrm{~m}$ length with $20 \mathrm{~cm}$ interplant distance, in the experimental field of Varamin Cotton Research Center of Iran, according to a completely randomized design (CRD) with 3 replications.

For cytogenetical studies, 50 flower buds were used randomly from 10 randomly selected

*Corresponding author, e-mail: msheidai@cc.sbu.ac.ir,msheidai@yahoo.com 

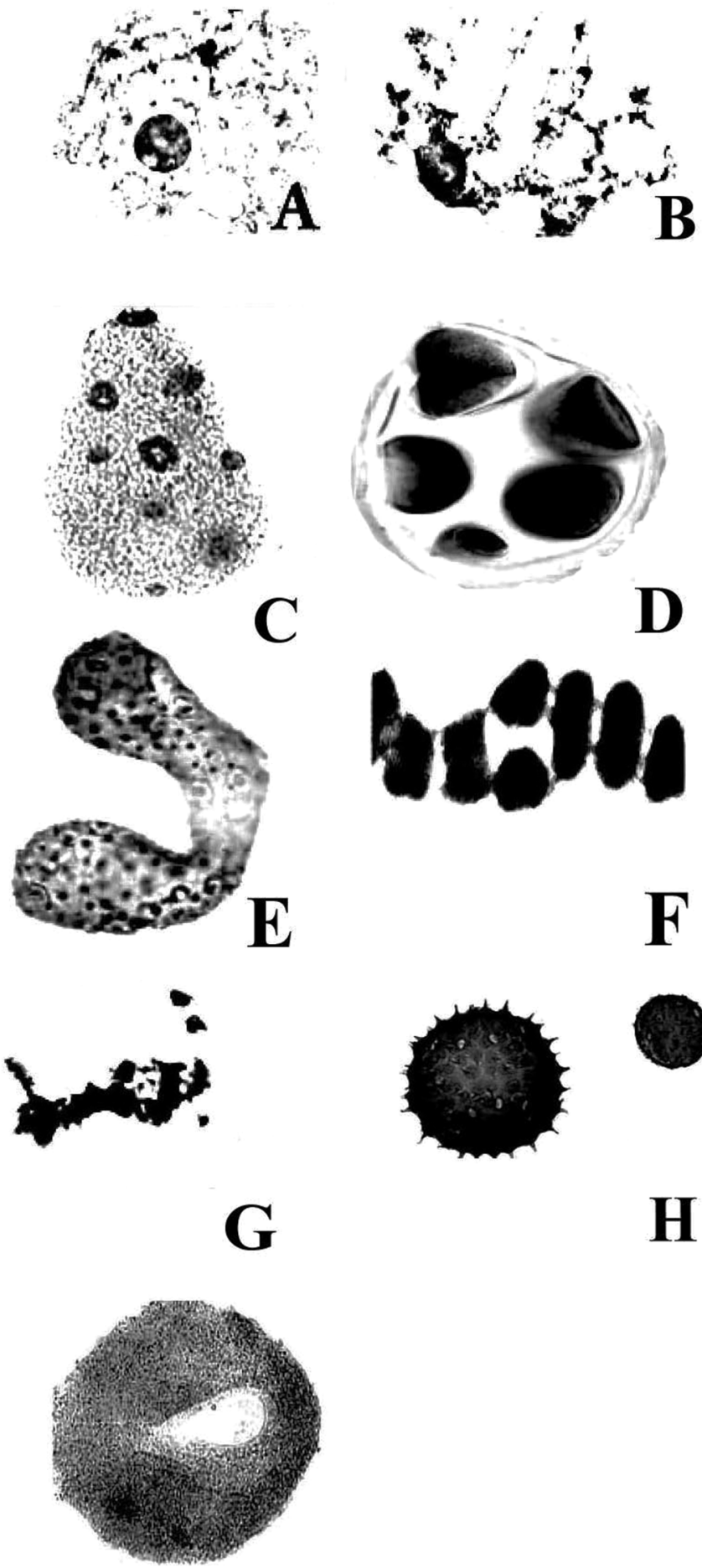

H 
Table 1. Cytogenetic characteristics of cotton cultivars studied (All values are the mean number)

\begin{tabular}{llllllllll}
\hline \hline Cultivar & TX & IX & TOX & RB & ROD & VI & IV & I & PF \\
\hline Var-76 & 45.40 & 4.03 & 46.33 & 21.30 & 3.20 & 0.00 & 1.00 & 4.00 & 97.60 \\
T-300 & 37.65 & 3.45 & 40.60 & 16.80 & 0.65 & 0.35 & 1.20 & 0.45 & 96.50 \\
S-150 & 43.95 & 1.25 & 44.20 & 21.65 & 2.40 & 5.00 & 0.15 & 0.00 & 96.20 \\
C-250 & 39.85 & 2.95 & 42.25 & 17.95 & 1.85 & 5.00 & 0.95 & 0.25 & 95.90 \\
C-200 & 29.80 & 1.85 & 31.55 & 14.35 & 1.75 & 0.15 & 0.05 & 0.00 & 96.00 \\
\hline
\end{tabular}

Abbreviations: $\mathrm{TX}=$ Terminal chiasmata, $\mathrm{IX}=$ Intercalary chiasmata, $\mathrm{TOX}=$ Total chiasmata, $\mathrm{RB}=$ Ring bivalents, $\mathrm{ROD}=$ Rod bivalent, $\mathrm{VI}=$ Hexavalent, $\mathrm{IV}=$ Quadrivalent and $\mathrm{I}=$ Univalent, $\mathrm{PF}=$ Pollen fertility.

plants of each line making the total collection $50 \times 10 \times 10=5000$. Squash technique used and pollen fertility test followed the earlier report (Sheidai et al. 1998). For morphological studies 5 plants were selected randomly from each genotype and their characters were noted down.

In order to determine a significant difference in cytogenetic characteristics as well as morphological characteristics the analysis of variance (ANOVA) followed by the least significant difference test (LSD) as well as $\chi^{2}$ test were performed among the genotypes. The grouping of the cultivras was performed by using different clustering methods as well as ordination based on principal components analysis (PCA). For cluster analysis, standardized data $($ mean $=0$, variance $=1)$ and Euclidean as well as squared Euclidean distances were used. Statistical analysis used SPSS ver. 9 (1998).

\section{Results and discussion}

\section{Diffuse stage in meiosis-I prophase}

The results of cytogenetic analyses are presented in Table 1 and Figs. 1-3. The meiotic analysis of all cotton lines studied showed the occurrence of a post pachytene diffuse stage (Fig. 1, A and B). Despiralization of chromosomes occurred after pachytene, commencing diffuse stage. The occurrence of diffuse stage has been reported in several plant species (Sybenga 1992). Diffuse may be of complete type in which the whole chromosomes decondense or it may be partial in which some parts of the genome show decondensation. The present study showed the occurrence of partial/complete diffuse in irradiated cotton lines. Various reasons have been suggested for the occurrence of diffuse stage, including high synthetic activity analogous to the lampbrush stage in amphibian oocyte, meiotic arrest to withstand the adverse environmental conditions, etc. (Sheidai and Inamdar 1991). However at present we do not know the reason for the occurrence of diffuse stage in cotton.

\section{Chiasma frequency and chromosome pairing}

The cytogenetic characteristics of the genotypes studied including chiasma frequency and distribution as well as chromosome pairing are presented in Table 1. The highest mean values of total and terminal as well as intercalary chiasmata occurred in the cultivar Var-76 (46.33, 45.40 and 4.03, respectively), while the lowest mean values of total and terminal chiasmata occurred in the cultivar C-200 (31.55 and 29.80, respectively). The lowest mean value of intercalary chiasmata occurred in the cultivar S-150 (1.25).

Fig. 1. A and B: Pachytene and post pachytene diffuse stage in hybrid cultivar C-150. C: Cytomixis between two neighboring meiocytes in the cultivar C-250. D: Meiocyte showing extra chromosomes due to cytomixis in the cultivar Var-76. E: Multipolar cell in the cultivar C-200. F: Abnormal tetrad in the cultivar T-300. G: A meiocytes showing vacuole like structure in the cultivar S-150. H: Abnormal pollen grain in the cultivar Var76. I: Potential unreduced pollen grain (large size pollen) in the cultivar C-200. Scale bar indicates $10 \mu \mathrm{m}$. 
Cultivars S-150 and Var-76 possessed the highest values of ring bivalents (21.65 and 21.30, respectively) while the cultivar C-200 possessed the lowest value (14.35). Adjacent and alternate quadrivalents were formed regularly in all the cultivars studied with the cultivar T-300 showing the highest value (1.20) and cultivar C-200 possessing the lowest value (0.05). Hexavalents occurred in all the cultivars studied except the cultivars Var-76 (Table 1).

The irrdaiated lines of the cultivars studied differ significantly in chromosome pairing compared to their normal nonirrdaiated cultivars. For example S-150 obtained from Sahel cultivar, shows 44.20 mean total chiasmata, 43.00 terminal and 1.25 intercalary chiasmata while these values in Sahel cultivar are 40.50, 38.24 and 2.32, respectively (Sheidai and Koobaz 2003). Moreover no hexavalent is formed in nonirradiated Sahel cultivar while, S-150 forms 0.05 hexavalents. ANOVA and LSD tests performed on chromosome pairing and chiasma frequency revealed a significant difference among the cultivars studied for the number of total and terminal chiasmata, the number of ring and rod bivalents. Such meiotic features are under genetic control, therefore the presence of a significant difference among the cultivars studied may indicate partly their genomic differences, as they were grown under uniform conditions.

\section{Meiotic abnormalities}

Chromosome stickiness occurred in all the cultivars studied, ranging from stickiness among two chromosomes to the involvement of all metaphase chromosome forming a complete clump. The highest percentage of metaphase I stickiness was observed in C-200 followed by T-300 (5.70 and 4.51, respectively), while no metaphase-I stickiness occurred in C-150 cultivar. The metaphaseII stickiness was observed in 2 cultivars of C-150 and C-250 (4.80 and 5.00\% respectively). Anaphase-I stickiness occurred in 3 cultivars of C-200, T-300 and Var-76. Genetic, environmental factors and genomic-environmental interaction have been considered as the reason for chromosome stickiness in different plant species (Baptista-Giacomelli et al. 2000).

Laggard chromosomes occurred in anaphase I and II ranging from 1 to a few chromosomes. The highest percentage of anaphase-I laggards occurred in Var-76 cultivar (18.75) while, no laggards occurred in anaphase-I of C-150 and C-250 cultivars. However the cultivars C-150, C-250 and C-200 showed the occurrence of anaphase-II laggards. Multipolar cells were observed in all the cultivars studied (Fig. 1, C) which may be the reason for abnormal tetrads and pollen grains formation in the cotton cultivars (Fig. 1, D and E). Such abnormality may lead to the formation of aneuploid cells and chromosome mosaics as observed in some grass species which is considered to be geneticaly controlled (Nirmala and Rao 1996). Pair-wise $\chi^{2}$ test performed showed a significant difference in the percentage of cells showing meiotic abnormalities among the cultivars studied.

\section{Cytomixis}

Cytomixis (chromosome/chromatin migration) was observed in all the cultivars studied (Fig. 1, F). Chromosome migration occurred in different directions from early prophase to late telophase II. Several metaphase cells showed the presence of extra chromosomes with different degree of compactness compared o the rest of chromosomes possibly due to cytomixis (Fig. 1, G). Several deformed and infertile pollen grains were also observed possibly due to cytomixis.

Migration of chromatin material among the adjacent meiocytes occurs through cytoplasmic connections originated from the pre-existing systems of plasmodesmata formed within the anther tissues. The plasmodesmata become completely obstructed by the deposition of callose, but in some cases they persist during meiosis and increase in size forming conspicuous inter-meiocytes connections or cytomictic channels that permit the transfer of chromosomes (Falistocco et al. 1995). Cytomixis has been reported in several plant species leading to the formation of aneuploid as well as polyploid meiocytes. Cytomixis leads usually to aneuploidy and reduction in fertility of plants, therefore it is considered to be of less evolutionary significance, however it may bring about new 


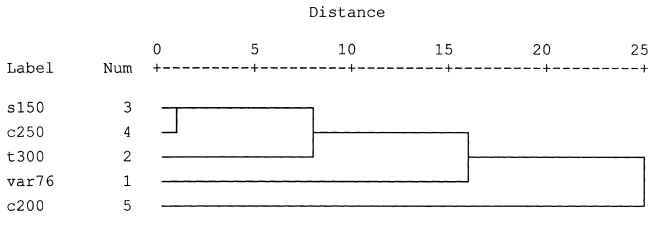

Fig. 2. UPGMA clustering of cotton cultivars based on cytogenetic characters.

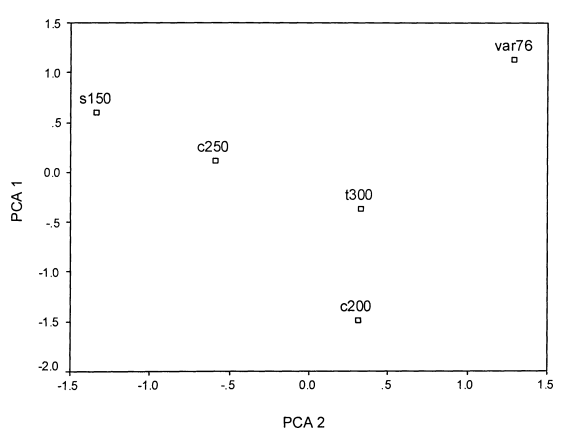

Fig. 3. PCA ordination of cotton cultivars based on cytogenetic characters.

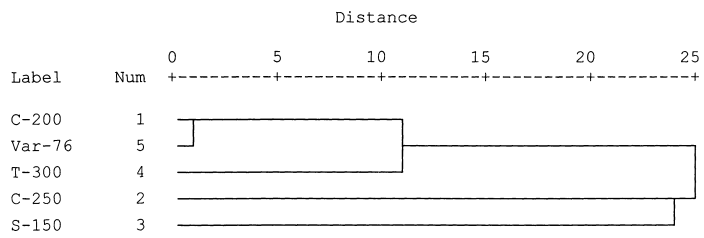

Fig. 4. UPGMA clustering of cotton cultivars based on morpho-agronomical characters.

Table 2. Morphological and agronomical characteristics of the cotton cultivars studied

\begin{tabular}{lcccccc}
\hline \hline Cultivar & $\mathrm{P}$ & $\mathrm{S}$ & $\mathrm{N}$ & $\mathrm{D}$ & $\mathrm{V}$ & $\mathrm{SN}$ \\
\hline C-200 & 2.78 & 16.22 & 6.20 & 19.44 & 4.60 & 19.77 \\
C-250 & 2.44 & 16.77 & 5.55 & 15.88 & 3.77 & 17.33 \\
S-150 & 1.11 & 21.00 & 5.88 & 20.44 & 3.00 & 17.77 \\
T-300 & 2.00 & 12.66 & 5.55 & 18.66 & 3.66 & 20.00 \\
Var-76 & 2.22 & 16.22 & 6.00 & 21.66 & 4.11 & 21.44 \\
\hline \hline Cultivar & $\mathrm{H}$ & $\mathrm{E}$ & $\mathrm{PW}$ & $\mathrm{Y} 1$ & $\mathrm{Y} 2$ & $\mathrm{~T}$ \\
C-200 & 97.77 & 2.20 & 20.38 & 4632.06 & 3708.43 & 8340.50 \\
C-250 & 88.33 & 2.20 & 22.27 & 5218.60 & 4137.19 & 9355.78 \\
S-150 & 99.66 & 2.03 & 20.60 & 3594.10 & 3592.81 & 7186.87 \\
T-300 & 110.00 & 2.58 & 21.64 & 5316.22 & 2510.00 & 7826.22 \\
\hline
\end{tabular}

Abbreviations: $\mathrm{P}=$ Pubescence, $\mathrm{S}=$ Length of the first sympodial branches, $\mathrm{N}=$ number of nodes till the first sympodial branch, $\mathrm{D}=$ Distance of the first sympodial branch to the ground level, $\mathrm{V}=$ number of vegetative branches, $\mathrm{SN}=$ number of sympodial branches, $\mathrm{H}=$ Plant height, $\mathrm{E}=$ Earliness \%, PW=Weight of pod, $\mathrm{Y} 1=$ First yield, $\mathrm{Y} 2=$ Second yield, $\mathrm{TY}=$ Total yield $\left(\right.$ all $\left.\mathrm{Kg} \mathrm{ha}^{-1}\right)$.

genetic variability by producing aneuploid gametes and new phenotypic characters as reported in other plants (Falistocco et al. 1995). In some cases cytomixis may lead to the migration of the whole chromatin material among the neighboring meiocytes and formation of unreduced gametes. The presence of giant pollen grains has been used as an indication of the production of $2 n$ pollen. It is interesting to mention that in the cultivars C-250, C-200 and T-300 some of the pollen grains formed were bigger in size, ranging from $50-70 \mu \mathrm{m}$ in diameter (Fig. 1, H), significantly $(p<0.01)$ larger than the rest of pollen grains (ranging from $40-50 \mu \mathrm{m}$ in diameter). These larger pollen grains may be considered as the potential $2 n$ (unreduced) pollen grains. 
The pollen fertility of the cultivars studied ranged from 95.90 to $97.60 \%$ (Table 1.) The correlation test performed among pollen fertility and meiotic abnormalities revealed a negative significant correlation between pollen fertility and total percentage of cytomixis, particularly with cytomixis at metaphase-I $(r=-0.77, p=0.05)$. Therefore cytomixis may be partly responsible for reduction in pollen fertility in the cotton lines studied.

The occurrence of vacuole like space was observed in the meiocytes of gamma-irradiated cottons studied (Fig. 1, I). This vacuole like space started by forming a tiny space inside the cell, growing and becoming larger till almost entirely fill up the whole cell. We do not know at present the reason for the formation of such a structure inside the meiocytes but it may also lead to the pollen infertility observed in the cultivars studied.

Different methods of cluster analysis as well as ordination of the irradiated cotton lines produced similar results (Fig. 4). The cultivars S-150 and C-250 show cytogenetic similarities and are placed close to each other, while the cultivars T-300 and Var-76 join them with some distance. The cultivar c-200 stands alone in a separate cluster showing its cytogenetic difference with the other cultivars studied. The separation of C-250 and C-200 in different clusters/groups is interesting since both cultivars are produced from Shirpan cultivar by using different gamma doses. These cultivars also stand in different clusters/groups based on morphological characters (Fig. 4), therefore it seems that different gamma doses have different effects on the same genotype i.e. Shirpan cultivar.

Factor analysis of meiotic characters revealed that the first 2 factors comprise about $84 \%$ of total variance. In the first factor with about $58 \%$ of total variance, meiotic characters including number of rod bivalents, total and terminal chiasmata possessed the highest positive correlation $(>0.90)$, while the number of quadrivalents possessed the highest negative correlation $(>-0.85)$. This factor separates mainly S-150 from the other cultivars (Fig. 3). Factor 2 comprises about 26\% of total variance in which the number of univalents and intercalary chiasmata possessed the highest positive correlation $(>0.70)$, while the number of hexavalents possessed the highest negative correlation $(>-0.89)$. This factor separates mainly the cultivar Var-76 from the others. Therefore these are the most variable meiotic characters among the irradiated cotton cultivars studied.

\section{Morpho-agronomical studies}

Morphological and agronomical characterstics of the cultivars studied are presented in Table 2. ANOVA and LSD tests showed the presence of a significant difference in all morphological characters among the cultivars studied. Different clustering methods as well as PCA ordination of the cultivars based on morpho-agronomic characters produced similar results (Fig. 4). In general 3 major clusters/groups are formed. Three cultivars of C-200, Var-76 and T-300 show more similarity in morphological characters and form the first major cluster while, C-250 and S-150 cultivars each stand separate from the other cultivars. The result of morpho-agronomical grouping agrees in part with that of cytogenetical clustering, for example separation of C-200 and C-250 and similarity between C-200 and T-300. In general both cytogenetical and morpho-agronomic analyses indicate distinctness of the gamma irradiated cultivars in their genotypic changes due to irradiation, which may be used in further breeding and selection in cotton.

\section{References}

Abo-Hegazi, A. M. T. and Shaheen A. M. 1988. Induced mutation and molecular techniques for crop improvement. Use of mutations to improve cotton plants as an oil and protein source without affecting the seed cotton yield. IAEA-SM. 311/78 P. 183-187.

Baptista-Giacomelli, F. R., Pagliarini, M. S. and Almeida, J. L. 2000. Meiotic behavior in several Brazilian oat cultivars (Avena sativa L.). Cytologia 65: 371-378.

Falistocco, E., Tosti, T. and Falcinelli, M. 1995. Cytomixis in pollen mother cells of diploid Dactylis, one of the origins of $2 n$ gametes. J. Heredity. 86: 448-453. 
Nirmala, A. and Rao, P. N. 1996. Genetics of chromosome numerical mosaism in higher plants. Nucleus 39: 151-175.

Sheidai, M. and Inamdar, A. C. 1991. Variations in meiotic chromosomes in Asparagus L. Biovigyanum 17: 45-47.

—, Vafai-Tabar, M., Mirzai-Nedoshan, H. and Hosseini-Nejad, Z. 1998. Cytogenetical studies in Gossypium hirsutum L. cultivars and their hybrids. Cytologia 63: 41-48.

- and Koobaz, P. 2003. Cytogenetic and morphometric study of some gamma irradiated tetraploid cotton cultivars. Nucleus 46: $20-28$.

Sybenga, J. 1992. Cytogenetics in Plant Breeding. Spring-Verlag, Berlin, Germany. 